\title{
An Investigation into the Nature of Mentoring Support in the Development of Teachers
}

\author{
Sharon Jenkins Carter
}

\begin{abstract}
The starting point for this research was an interest in how people learn and develop as adults and, more specifically, how people can help each other, either consciously or perhaps unconsciously, to develop on both a professional and personal level. Society is structured so that individuals live and work together within a framework of cooperation and the focus here is the role of this framework in vocational development. Colleagues undoubtedly have a role to play in the development of others. Some willingly offer advice, assistance or just listen where needed; others may be more reluctant to do so, perhaps afraid of revealing their own vulnerability. There is a sense that it's not so much that we live in completion with each other, but in ignorance of how we might support each other.
\end{abstract}

Index Terms-Mentoring support, teachers, adults, learn and develop.

"Everyone who makes it has a mentor" (Collins and Scott, 1978) [1]

\section{INTRODUCTION}

On both a professional and a personal level, people are the best possible resource for our further development. In organizational terms, the increasing pressures of globalization and the pace of technological innovation are forcing organizations to the realization that people from their most potent resource and hence give them the competitive edge. As a result, the approach to the twenty first century is characterized by increasing commitment to the issues of human resource development. How can people be most effective at work? What is it that ensures their commitment to common goals? Does interest in personal development have a positive effect on professional development? These are some of the issues raised in the field of Human Resource Development.

One of the areas of Human Resource Development which is of interest to me, personally, is Mentoring. It is more than a method of training individuals in a particular skill. It represents a different approach to the way in which individuals can be helped to explore their potential. Mentoring is important as a technique, in that it is concerned with the development, not just of the individual in the work place, but also with the personal side of human development. In promoting mentoring, its advocate are not focusing on the development of a limited set of skills but on

Manuscript received February 17, 2013; revised May 17, 2013.

Sharon Jenkins Carter is with the Derbyshire County Council, United Kingdom (e-mail: sharon.n.carter@hotmail.co.uk). a broader perspective that relates to the whole of an individual's relationship to work and his/her ability to thrive within it. It is the complexity and depth of the relationship which makes it such an exciting proposition in terms of human resource development:

"Being involved in a mentoring relationship can help put your life in perspective. The experience can often develop and reveal parts of yourself that you never knew existed." (Sylvia Ashley 1994 P33) [2]

It has been described as a journey (Daloz 1983) [3] in which the mentor acts as a guide and points the way; at the same time, offering support and challenges to the protégé. The nature of the relationship is such that a commitment to learning and development should surely operate alongside the fundamental social issue of taking an interest in others, and being able and prepared to offer assistance when the occasion arises.

In view of this, I wanted to find out how sensitive teachers are to the needs of their own colleagues; whether or not the learning environment which they themselves help to create, extends to colleagues as well as to children. My research examined the quality and features of colleague support given to teachers, in relation to their personal and professional development. It addressed the question: to what extent could this support be termed mentoring and to what extent could it be related to any of the models of mentoring which have been produced by previous researchers?

\section{WHAT IS MENTORING?}

Even though the term 'mentoring' is receiving a great deal of attention, its origins can be traced back to Greek mythology when Ulysses left the tutelage of his son, Telemachus, in the hands of his friend, Mentor. Clutterbuck (1985) [4] traces modern day mentoring back to the apprenticeship system, when the apprentice learnt his skills from the master craftsman.

Today the term "Mentoring" conjures up several different images. Clutterbuck (1985) [4] identifies four: it may refer to the development of promising young talent in an organization; it may mean the facilitation of young people into an organization; it may be an effective means for women to achieve a higher profile in an organization; or it could be viewed as favoritism, open to all the pressures such a relationship may cause.

These various images reflect the elusive nature of an exact definition of the term "Mentoring". However, it usually refers to a one-to -one relationship between a senior and a more junior member of an organization, where one person offers guidance, help, support and advice to facilitate the learning or development of the other. 
Known as "Classical Mentoring", this definition is supported by the work of Kram (1985) [5], Levinson (1978)[6], Roche (1979) [7]. It is characterized by the fact that the two people involved gravitate towards each other. However, in recognition of the successful aspects of informal mentoring, a formal system of mentoring which involves a structured relationship has evolved, where the protégé is assigned to a mentor. If it is to be effective, it should involve considerable emotional commitment on both sides. (Clutterbuck 1985) [4]. The success of such formal schemes has been challenged by several researchers (Chao, Waltz, Gardner 1984, Kram 1985, Caruso 1989) [8], [5], [9]. There may be personality conflicts in an assigned relationship, or a lack of commitment from either party could hinder the development of mentoring functions.

"Informal mentorships arise because of a desire on the part of the mentor to help the protégé and a willingness on the part of the protégé to be open to advice and assistance from the mentor". (Chao, Waltz, Gardner, 1992) [8].

Caurso's study of an established formal mentoring scheme at Motorola (1989) [9]. In fact threw considerable light on the effect of informal mentoring relationships. His research revealed that a protégé's professional and emotional support came from, not just one, but from a number of sources and that the assigned mentor did not figure as the main protagonist.

"Our research at Motorola,... determined that the formally assigned mentor, although heavily resourced, was not the protégé's primary source of mentoring help. Equally important is the fact that no single individual supplied most of the help received by the protégé; in other words, protégés did not simply substitute a naturally selected mentor for the one designated by the company." (Caruso) [9].

Caruso did point out that the culture of the organization has an important influence on determining both the nature and the effect of the mentoring relationship. As a result of his study, he describes a different perspective on a mentoring relationship as:

"The collective source from which a protégé seeks and receives mentoring help constitutes the protégé's mentor." (Caruso) [9].

Kram (1985) [5] refers to these collective sources as "relationship constellation."

The impetus falls on the character and needs of the protégé in this "open system" of mentoring, and the success could be seen as a product of these needs to "structure and manage" the mentoring relationship. (Caruso 1991)[10]. Social skills, effort and availability of resources are all cited as criteria for success.

"The success of the protégé is a function of the needs of the protégé, the effort put forth to obtain help, the availability of sources of mentoring help and the social skills of the protégé in finding and negotiating such help." (Caruso) [10].

Research by Kram (1985) [5] has identified two main areas of support provided by the mentoring relationship: career development and psychosocial support. Career functions include sponsorship, visibility, exposure, coaching, protection, challenging assignments. Psychosocial functions include such aspects as role-modeling, friendship, counseling, acceptance and confirmation.
Another aspect of mentoring is the mentoring relationship between peers. This is an informal relationship between two or more colleagues, with an emphasis on equality of status. It may well be that an adult is involved in several peer relationships at the same time, each one possibly fulfilling some aspect of mentoring, but together, forming a collective resource as suggested above by Caruso. Kram and Isabella (1985) [11] have identified a 'continuum of peer relationships': Information Peer; Collegial Peer; Special Peer. The nature and level of support in these relationships begins with the sharing of information and then moves on to involve a greater level of trust and friendship. Another feature of peer mentoring is the two-way process. In sharing their wisdom or knowledge with each other, individuals can 'feel like experts while learning and deriving support from a colleague'. (Kram) This adds another aspect to the psychosocial functions discussed earlier.

\section{ARGUMENT}

My initial starting point, the level of support that teachers receive from colleagues in terms of their professional development, has led me to recognize that this development cannot be divorced from the personal map, or history, of individual teachers involved. Implicit in this approach is the idea that each teacher brings a slightly different angle to the concept of teaching, determined by previous knowledge, ideas and perceptions of the profession. The very personal nature of teaching is reflected in the variety of styles to be found in the classroom and in the extent to which the teacher has to draw on his/her own personality. This is the process referred to by Fuller and Brown (1975) [12] as "constant, unremitting self-confrontation."

From the outset, I was interested in trying to paint a picture which would highlight this personal process whilst at the same time would allow for some common themes to emerge in a study of how teachers actually support each other in their professional development and what form that support takes. I was centrally interested in finding out whether or not the various levels of support that teachers receive from one another, correspond to any of the models of mentoring discussed earlier, in particular the informal mentoring, perhaps supplied by a heat or senior teacher, and peer mentoring.

I was therefore concerned that my research tool should allow for the emergence of such a rich and diverse picture and should take into account individual biographies and histories. This subjective approach which allowed the individuals to present their own perspective on their development, offers a relative view, a phenomenological approach, which allows for the uniqueness of individual experience to be portrayed in a multi-faceted view on the nature of professional support in teacher development. Hence the choice of qualitative research, to quote Judith Bell: "Researchers adopting a qualitative perspective are more concerned to understand individuals' perceptions of the world. They seek insight rather than statistical analysis." (1987) [13].

I interviewed ten teachers, nine of whom were head teachers and the other person a part-time class teacher. I decided to include the latter in case the story of someone 
who had not, according to the promotional criteria in teaching, yet achieved such success, had a different emphasis or would reveal some completely different aspect.

\section{A. Overview of Findings}

Analysis of the data revealed three significant themes concerning the nature of teaching support:

- The role of the head teacher;

- The role of colleagues;

- The ethos of the school.

Obviously these three themes do interlink and overlap at certain points. The ethos of the school depends on the vision of the head teacher and the commitment of colleagues. For the purpose of clarity, the head teacher is presented by the data as providing a figurehead and, at the same time, as the founder or the vision behind the culture of the school; the role of colleagues is similarly two-fold in that they are identified as providing a range of specific supportive techniques, whilst at the same time, their commitment to the culture of the school is vital for its continuance. Fig. 1 Together these themes provide a whole range of supportive techniques: from practical knowledge to gestures of reassurance. The data also revealed that this support appeared critical in providing the necessary foundations for self-motivation.

\section{School Ethos, Head teacher, Colleagues Support \\ $\sqrt{3}$ \\ Motivation}

Fig. 1. Illustrates relationship between support and motivation

Not everyone spoke of strong support in all three areas at the same time, but it would appear that they all identified a significant time in their professional career when a combination of the above themes existed, with sufficient impact to provide them with a "kick-start" in selfmotivation and development in general.

From this a fourth category of results emerged informing understanding of the area it identifies the stance of the interviewees themselves in responding to this support;

\section{B. Motivation and Self-Esteem}

Many of the interviewees spoke of how support enabled them to grow in confidence and take control of their own development. Confidence, self-esteem, self-efficacy are all factors which emerged as contributory to this process of personal and professional development. All of those interviewed spoke of a particular colleague as a friend, who fulfilled some of Kram's psychosocial and career mentoring functions, and at the same time could also identify other colleagues from whom some aspect of mentoring support was forthcoming. All of the interviewees spoke of the need for support, by either consciously asking, or subconsciously looking, and this would seem to suggest Caruso's model of mentoring with the protégé at the centre of a number of relationships which collectively fulfill the mentoring function.

As well as colleague support, they all mentioned the role of the head teacher as a vital factor in securing their commitment and motivation. The word 'inspiring' appeared frequently in describing this figure, accompanied by a level of personal interest shown and the ensuing confidence which the teacher drew from this.

They all mentioned that the head took a personal interest in their professional well being and all commented on the high standards and expectations of their head teacher and felt that these values not only touched on something within them, but also, that they were values which they carried during their career. It appeared that they witnessed behaviors in their head teachers which they regarded as inspiring: ability to motivate, knowledge of educational theory and practice, personality and leadership qualities, judgment and trust. They all expressed great respect for these head teachers and recalled that their professional development was considerable in terms of being motivated, by these behaviors, to develop a lasting and committed interest in education. This relationship provided, at the very least, a subset of the mentoring functions detailed by Kram, and corresponds to the traditional view of the mentor being a more senior, and usually, an older member of an organization.

The evidence of the interviewees also pointed to the wide range of professional and personal support from colleagues. The personal support on offer was both wide and deep for all of the interviewees. The evidence was substantial and would certainly seem to lend itself to the idea that peer support is vital amongst primary school teachers, that this support is extensive in range and is recognized as a significant factor in individual development.

"Creating a mentoring culture enables an organization to enrich the learning that takes place throughout the organization." [14]

The support seemed to be very much a two-way thing, with interviewees seeking help from a variety of sources, from a number of colleagues at the same time, and this is in keeping with Caruso's notion of mentoring in which the individual seeks help from a number of sources. Caruso's model is firmly set with the individual at the centre and draws upon the character and needs of this person as the criteria for its success. 'Character', in this instance, refers to a level of motivation and so brings up the question of personal development and its elusive relationship with professional development. This view that mentoring is driven by an individual as the agent of the mentoring support is reflected in the pro-activity of many of the interviewees in seeking support, recognizing and responding to what was being offered. Thus the theme of the interviewee as the agent of their own mentoring was implicit in most of the interviews. As Steve Blank writes:

"A mentor relationship is a two-way street. To make it work, you have to bring something to the party." [15]

Similarly, Eric Parsloe identifies self- awareness as critical in a mentor relationship:

"Coach -mentoring can only start when learners develop an awareness of the need and desire to improve their performance or change the way they have been doing things at work as well as in life generally. Without a genuine recognition of need and desire, it is almost impossible to change behavior." Parsloe (2009) [16]

Arising from both the behaviors of the head teacher and the colleagues was an important area of support in the 
development of teachers which might be termed collective values and behaviors or school ethos. Out of this arose a commitment to those educational ideals and the result was a staff who worked together in their search for quality. The work of Nias (1989) [17], emphasizing the link between values and commitment to development was clearly supported. The challenge of the job and the blending of attitudes and ideas created a culture of trust and mutual respect, in which staff worked together, talked with each other, in pursuit of further goals. Burley and Pamphrey (2011) talk about a space "... where teachers can develop a shared focus and purpose for their professional learning." [18]

It could be argued that the evidence showed that elements of peer mentoring could be identified in a culture where staff were committed to the vision and had a fundamental belief in what they were doing. The individuals were able to identify strongly with the group and with the collective beliefs and along with colleagues, reflect upon their experiences. Did long-term learning have its origins in such a positive environment? It would appear to be the case from analysis of the data but further research would be needed to substantiate this.

Analysis of the data also revealed a close relationship between confidence and levels of motivation. Praise, feeling valued, recognition from others are all recurrent themes which promoted confidence. This in turn, led to a re examination of feelings and a self awareness which awakened either a belief in the professional mission or confirmation of personal qualities. This confidence could therefore be read as personal growth. This self-efficacy (Bandura) [19], the belief one has in one's own ability to deal effectively with the environment, would appear to be directly related to those who seek mentoring support, in that those with higher self-efficacy are more likely to seek ways of changing their circumstances if they felt the need to do so. Keller echoes this:

"In general, a healthy sense of self-efficacy, promotes greater effort and perseverance, higher standards for performance and higher expectations for positive outcomes." (2012) [20]

Positive feedback from mentoring support would have an effect on one's own level of confidence and this, in turn, would raise one's own level of self-efficacy. So it would appear to follow that there is a link between professional and personal development and that mentoring functions play a vital role in the nature of this relationship.

\section{CONCLUSION}

\section{A. Finally, Some Thoughts}

Nobody indicated that they entered the profession with great personal ambitions. However, of those who tried to reflect on a feeling of awakening in this respect, they referred to a 'culture of collaboration' as being particularly conducive. Fig. 2

Would those interviewed have been successful in terms of career development without experiencing such an ethos of commitment?

\section{Belief in Values and Ethos of School<smiles>CCCCC[AsH2][AsH2][SiH3]</smiles>

Staff worked together

Staff presented goals for each other

Fig. 2. Collaboration reinforces commitment to common values, underpinned by ethos.

Further research into this would have particular significance for the development of mentoring schemes.

With support and thereby increased confidence, a sense of harmony between public and private self appeared to emerge and, upon this basis, a certain readiness for change or development. This could be represented as follows:

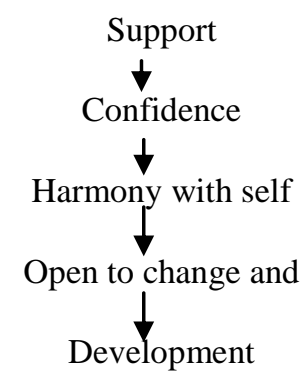

Fig. 3. The effect of mentoring support on personal and professional development

It would be interesting to research further into the exact nature of the relationship between support and confidence.

Is development dependent upon attaining a certain level of confidence?

Drawing these threads together, it would appear that Bandura's notion of self-efficacy has a place in this discussion. As discussed earlier, the belief one has in one's ability to deal effectively with the environment is the basis for actually dealing effectively with it: self-activism. This self-activism could be interpreted along the lines of Caruso's protégé-driven system of mentoring relations: the individual actively seeking mentoring support. This could be illustrated as follows:

\section{Positive feedback
Confidence
Self-efficacy
Self-activism
Mentoring Support}

Fig. 4. The relationship between self-confidence and levels of mentoring support

The mentoring relationship provides positive feedback which, in turn, leads to an increase in level of confidence. However, it should be noted that this is only a hypothesis and would need to be the subject of further research.

To conclude, Daloz called mentoring 'a journey' in which 
the mentor guides the protégé along the way 3. Perhaps the main point here is that this research is only part of that journey, but it would be hoped that it has paved the way for furthering discussion of the issues raised.

\section{REFERENCES}

[1] E. Collins and P. Scott, "Everyone who makes it has a mentor," Harvard Business School Review, vol. 56, no. 4, 1978.

[2] S. Ashley, "Roots and wings," A Multi-Pronged Approach to Mentoring Tutoring, vol. 2, no. 2, Autumn, 1994.

[3] Daloz, "Mentors: Teachers who make a difference," Change, vol. 15, no. 6, 1983.

[4] D. Clutterbuck, "Everyone needs a mentor: How to foster talent within an organization," IPM, 1985.

[5] K. E. Kram, "Mentoring at work," Glenview 111 Scott, Foresman, 1985.

[6] D. J. Levinson, C. N. Darrow , E. B. Klein, M. H. Levinson, and B. McKee, Seasons of a Man's life, New York: Knopf, 1978.

[7] G. Roche, "Much ado about mentors," Harvard Business Review, Jan. / Feb. Smirchich and Morgan, 1980.

[8] G. T. Chao, P. M. Waltz, and P. D. Gardner, "Formal and informal mentorships: A comparison of mentoring functions and contrast with no mentored counterparts," Personnel Psychology, 1992.

[9] R. E. Caruso, "Mentoring and the development of social capital," in Human Resource Management: People and Performance, ed., Bradley K. Dartmouth, 1989.

[10] R. E. Caruso, "Mentoring, a different perspective," London School of Economics, Business Performance Group, 1991.
[11] K. E. Kram and L. A. Isabella, "Mentoring alternatives: The role of peer relationships in career development," Academy of Management Journal, vol. 20, 1985.

[12] F. Fuller and O. Brown, "Becoming a teacher," in Ryan K, Teacher Education, $74^{\text {th }}$ Year Book of the National society for the Study of education, University of Chicago Press, 1975.

[13] B. Judith, Doing your research project, OUP, 1978.

[14] L. J. Zachay, Creating a mentoring culture: The Organization's Guide, Jossey-Bass, 2005.

[15] Steve Blank. [Online]. Available: http://steveblank.com/2011/04/19/mentors-coaches-and-teachers/

[16] E. Parsloe, "Coaching and mentoring: Practical conversations to improve learning," Kogan Page, 2009.

[17] J. Nias, Primary teachers talking, Routledge, 1989.

[18] S. Burley and C. Pamphrey, Mentoring and coaching in schools: Professional learning through collaborative inquiry, Routledge 2011.

[19] A. Bandura, "Social Learning Theory," Englewood Clifts, NJ Prentice-Hall, 1997.

[20] T. E. Keller, The blackwell handbook of mentoring: A multiple perspectives approach, Ed Allen and Eby, Wiley-Blackwell, 2010.

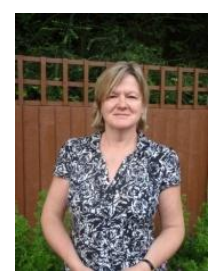

Sharon Jenkins Carter is a teacher and a language trainer in the UK. She has a French degree from Reading University, UK (1980) and a Masters Degree in training from Leicester University UK (1996). She has designed, written and delivered several language training courses for teachers in the UK. Sharon Jenkins Carter is particularly interested in mentoring and motivation in the workplace. 